\title{
Vasopressor treatment and mortality following nontraumatic subarachnoid hemorrhage: a nationwide electronic health record analysis
}

\author{
*George Williams, MD, ${ }^{1}$ Vahed Maroufy, PhD, ${ }^{2}$ Laila Rasmy, MSc, ${ }^{3}$ Derek Brown, PhD, ${ }^{2}$ \\ Duo Yu, MSc, ${ }^{2}$ Hai Zhu, PhD, ${ }^{2}$ Yashar Talebi, MD, ${ }^{2}$ Xueying Wang, MSPH, ${ }^{2}$ Emy Thomas, MS, ${ }^{2}$ \\ Gen Zhu, MSc, ${ }^{2}$ Ashraf Yaseen, PhD, ${ }^{2}$ Hongyu Miao, PhD, ${ }^{2}$ Luis Leon Novelo, PhD, ${ }^{2}$ \\ Degui Zhi, PhD, ${ }^{2,3}$ Stacia M. DeSantis, PhD, ${ }^{2}$ Hongjian Zhu, PhD, ${ }^{2}$ Jose-Miguel Yamal, PhD, ${ }^{2}$ \\ David Aguilar, MD, ${ }^{1,2}$ and Hulin $\mathrm{Wu}, \mathrm{PhD}^{2,3}$ \\ ${ }^{1}$ McGovern Medical School, ${ }^{2}$ School of Public Health, and ${ }^{3}$ School of Biomedical Informatics, University of Texas Health Science \\ Center, Houston, Texas
}

OBJECTIVE Subarachnoid hemorrhage (SAH) is a devastating cerebrovascular condition, not only due to the effect of initial hemorrhage, but also due to the complication of delayed cerebral ischemia $(\mathrm{DCl})$. While hypertension facilitated by vasopressors is often initiated to prevent $\mathrm{DCl}$, which vasopressor is most effective in improving outcomes is not known. The objective of this study was to determine associations between initial vasopressor choice and mortality in patients with nontraumatic SAH.

METHODS The authors conducted a retrospective cohort study using a large, national electronic medical record data set from 2000-2014 to identify patients with a new diagnosis of nontraumatic SAH (based on ICD-9 codes) who were treated with the vasopressors dopamine, phenylephrine, or norepinephrine. The relationship between the initial choice of vasopressor therapy and the primary outcome, which was defined as in-hospital death or discharge to hospice care, was examined.

RESULTS In total, 2634 patients were identified with nontraumatic SAH who were treated with a vasopressor. In this cohort, the average age was 56.5 years, $63.9 \%$ were female, and $36.5 \%$ of patients developed the primary outcome. The incidence of the primary outcome was higher in those initially treated with either norepinephrine (47.6\%) or dopamine (50.6\%) than with phenylephrine (24.5\%). After adjusting for possible confounders using propensity score methods, the adjusted OR of the primary outcome was higher with dopamine (OR 2.19, 95\% Cl 1.70-2.81) and norepinephrine (OR 2.24, 95\% Cl 1.80-2.80) compared with phenylephrine. Sensitivity analyses using different variable selection procedures, causal inference models, and machine-learning methods confirmed the main findings.

CONCLUSIONS In patients with nontraumatic SAH, phenylephrine was significantly associated with reduced mortality in SAH patients compared to dopamine or norepinephrine. Prospective randomized clinical studies are warranted to confirm this finding.

https://thejns.org/doi/abs/10.3171/2020.2.FOCUS191002

KEYWORDS subarachnoid hemorrhage; vasopressors; norepinephrine; phenylephrine; dopamine; mortality

$\mathrm{A}$ NEURYSMAL subarachnoid hemorrhage (SAH) is a potentially devastating cerebrovascular condition, due not only to the effect of initial hemorrhage but also to the complicated treatment regimen required to manage such patients. ${ }^{37}$ The incidence of SAH in the population has been estimated to be approximately 14.5 per 100,000 person-years, with a mean age of 55 years. ${ }^{18,34}$ As a result of the pathophysiology of this disease and its treatments, $25 \%-44 \%$ of patients who present with SAH die, and half of the survivors are left with some degree

ABBREVIATIONS ATE = average treatment effect; $\mathrm{CCI}=$ Charlson Comorbidity Index; $\mathrm{DBP}=$ diastolic blood pressure; $\mathrm{DCl}=$ delayed cerebral ischemia; EHR = electronic health record; EMR = electronic medical record; GBM = gradient-boosted model; GCS = Glasgow Coma Scale; HR = heart rate; IPW = inverse probability weighting; MI = myocardial infarction; PRES = posterior reversible encephalopathy syndrome; SAH = subarachnoid hemorrhage; SBP = systolic blood pressure.

SUBMITTED December 31, 2019. ACCEPTED February 14, 2020

INCLUDE WHEN CITING DOI: 10.3171/2020.2.FOCUS191002.

* G.W., V.M., and L.R. share first authorship of this work. 
of neurological deficit., ${ }^{4,511}$ The average age of onset for SAH may be young, although this particular type of stroke syndrome is associated with traditional risk factors such as hypertension, which predispose patients to active aneurysmal disease. ${ }^{36}$ Substantial resources are dedicated to currently accepted management paradigms, which include staffing by neurosurgeons, neurologists, neuroradiologists, neurointensivists, neuroanesthesiologists, and specialized nursing and rehabilitation personnel. Given the relatively young age of patients with SAH and associated disability, the health and economic burden to the individual can be devastating.

One of the complications of SAH that medical management seeks to avoid or minimize is delayed cerebral ischemia (DCI), as it is the main source of morbidity following SAH. ${ }^{32}$ Decreased cerebral perfusion (relative hypotension) and cerebral arterial vasospasm that may commonly occur after SAH are believed to be significant contributors to DCI. While $70 \%$ of patients with SAH show signs of radiographic cerebral vasospasm, approximately $20 \%-30 \%$ of SAH patients have clinical signs of cerebral vasospasm..$^{16}$ There are several proposed mechanisms contributing to cerebral vasospasm, including damage to the endothelium, smooth-muscle contraction, a change in vascular responsiveness, and inflammatory changes to the vascular wall. ${ }^{16}$ Besides the administration of nimodipine prior to clinical evidence of cerebral vasospasm, the avoidance of hypovolemia and hypotension is accepted as a mainstay of therapy when cerebral vasospasm is suspected. Induced hypertension to reduce the incidence of DCI was first described in the 1970s, and its benefit is clinically accepted by many neurointensivists. ${ }^{12}$ A systolic blood pressure goal of $160-180 \mathrm{~mm} \mathrm{Hg}$ is a widely accepted blood pressure target for SAH patient treatment following coil embolization of a cerebral aneurysm; this hemodynamic approach combined with avoidance of hypovolemia is commonly described as hypertensive hypervolemic therapy. ${ }^{8} 15$ Recent studies showed that vasopressor-induced hypertension is recommended over only a fluid regimen. ${ }^{29,33}$ Dopamine, norepinephrine, and phenylephrine are the most commonly accepted drugs to achieve the desired increase in blood pressure. ${ }^{30}$ Vasopressin is occasionally used, but is not generally accepted as a mainstay of therapy. ${ }^{23}$ While current guidelines ${ }^{8}$ suggest that maintaining an elevation in blood pressure when managing aneurysmal SAH may reduce the incidence of DCI, little clinical data exist to demonstrate which vasopressor is most efficacious to achieve induced hypertension and ultimately reduce mortality and other long-term adverse outcomes. In the absence of clinical trial data, the optimal vasopressor choice is based on multiple other factors, including the patient's hemodynamic status, comorbid conditions, and institutional preferences.

The Cerner Health Facts electronic medical record (EMR) database comprises de-identified electronic health record (EHR) data from over 700 participating Cerner client hospitals and clinics in the US, including patient demographics, encounters, diagnoses, procedures, laboratory results, medications, vital signs, and other clinical observations. ${ }^{6,28}$ The large population in this databaseapproximately 50 million patients-allows for performing in-depth studies on rare disorders with sufficient power to detect clinically meaningful effect sizes in which clinical trials may be difficult to perform. In this study, we queried the Cerner database to determine the association of vasopressor choice with inpatient mortality. We hypothesized that the choice of vasopressor is associated with mortality for SAH patients after adjusting for possible confounding factors.

\section{Methods}

\section{Study Design and Patient Population}

Following IRB approval from the University of Texas Health Science Center, we used the Cerner Health Facts EMR database, which consists of EMRs from more than 700 hospitals and clinics that use the Cerner Corporation's EHR system. The Cerner Health Facts EMR database (version 2015) includes approximately 50 million unique patients $(49,826,219)$, of whom 39,017 patients had at least one encounter with one SAH diagnosis identified by ICD-9 code $(430,800.2 x, 800.7 x, 801.2 x, 801.7 x, 803.2 x, 803.7 x$, 804.2x, 804.7x, 852.x) with 17,273 aneurysmal SAH cases. We excluded all patients younger than 17 years of age. A total of 4850 patients were administered at least one of the three vasopressors: dopamine, phenylephrine, or norepinephrine (National Drug Codes for included vasopressors are shown in Supplementary Table S1). If a patient had multiple SAH encounters within 24 hours of each other, we combined the encounters into a single encounter; otherwise, we only used the data from the first SAH encounter per patient in our analysis.

In this study, we examined the first vasopressor administered to eligible SAH patients. We excluded patients who initiated vasopressor treatment with two vasopressors simultaneously $(\mathrm{n}=40)$. Among the remaining $4810 \mathrm{SAH}$ patients, 3078 (64\%) were prescribed only one of the three vasopressors during the encounter period, 1437 patients $(30 \%)$ received a second vasopressor after the initial vasopressor, and 295 patients $(6 \%)$ were treated with all three vasopressors after the initial vasopressor. We grouped the patients into either dopamine, phenylephrine, or norepinephrine groups based on the initial vasopressor received, regardless of whether they subsequently received a different vasopressor. Among 4810 eligible patients with SAH, 2176 patients $(45.2 \%)$ had trauma-associated SAH (ICD9 codes 800.2x, 800.7x, 801.2x, 801.7x, 803.2x, 803.7x, 804.2x, 804.7x, 852.x) and 2634 patients had a nontraumatic SAH diagnosis. We analyzed SAH patients and nontraumatic SAH patients separately, which resulted in similar conclusions; this report will focus on nontraumatic SAH patients.

\section{Statistical and Causal Inference Analysis Methods}

The primary outcome was in-hospital mortality, defined as in-hospital death or discharge to hospice care. We calculated the Charlson Comorbidity Index (CCI) to provide an indicator of clinical morbidity across the populations. We also compared descriptive statistics for baseline, demographic, comorbidity, and outcome differences among the three treatment groups. For continuous variables, we used an ANOVA or Kruskal-Wallis test, depending on whether the ANOVA assumptions were met. For categorical variables, Fisher's exact test was used. 
Because patients with high-grade SAH have prolonged intensive care unit stays with multiple nosocomial complications such as pneumonia, anemia, infection/sepsis, renal failure, myocardial infarction (MI), and heart failure (cardiomyopathy), we compared proportions of these diagnoses among the treatment groups in a secondary analysis. Additionally, we included diabetes and liver disease as they are, in general, associated with worse outcomes. Furthermore, to account for patient severity, we included Glasgow Coma Scale (GCS) scores, which have been shown to be comparable to Hunt and Hess and World Federation of Neurosurgical Societies scale scores, and a significant factor for predicting severity at discharge. ${ }^{25}$

We used propensity score models to account for potential confounding variables and facilitate causal inference between vasopressor treatments and mortality. In a binary treatment case, the propensity score is the probability of receiving the treatment conditional on a given set of observed potential confounding factors. ${ }^{31}$ This probability can be calculated using standard regression techniques (typically logistic regression), with the treatment being considered the outcome and the potential confounding factors as the predictors. Treated and control subjects with similar estimated values for their propensity scores should have, on average, similar sets of covariate factors (Greene TJ, DeSantis SM, Swartz MD, unpublished data, 2017). We used inverse probability weighting (IPW) in the propensity score model, given its well-studied benefits. ${ }^{2,3}$ We used propensity score matching and generalized gradient-boosted models (GBMs), ${ }^{19}$ a machine-learning approach based on trees that selects predictive variables while higher-order variable interactions are automatically taken into account, to create the propensity score. Then we used this machine-learning approach to estimate the average treatment effect (ATE) inverse probability weights to balance treatment groups. We estimated propensity score vectors representing the probability of receiving each of three treatments (i.e., the generalized propensity score) using GBMs. The potential confounders we included in the propensity model are age, race, marital status, sex, GCS score, 750 medications (generic names) administered prior to the vasopressor treatment, CCI, and total intravenous fluid administered throughout the encounter. Also, to account for other complications, we adjusted for the 443 diagnoses that were present during admission across all patients. Prior to GBMs, we conducted variable selection using L1-penalized generalized linear models (GLM Lasso) ${ }^{26}$ so that the significant confounders associated with the choice of vasopressor treatment were identified. We then applied GBM to the selected variables, generated the inverse probability weights (where the weight is the inverse propensity of the treatment an individual actually received ${ }^{10,13}$ ) in weighted regression models,${ }^{19}$ and estimated ATEs of the vasopressor treatments on mortality.

We assessed the balance achieved through IPW by graphic representations (Supplementary Fig. S1). For each pairwise treatment comparison (dopamine vs phenylephrine, dopamine vs norepinephrine, and phenylephrine vs norepinephrine), we calculated a standardized mean difference before (unweighted) and after implementation of IPW. Then, for each selected confounder we graphed the maximum standardized mean difference across the three vasopressor treatment comparisons. From these plots, we evaluated the balance of potential confounders between the comparison groups.

\section{Sensitivity Analysis}

We conducted several sensitivity analyses to assess the robustness of these results. We used multiple logistic regression models to fit the outcome mortality and covariate vasopressors, as well as variables selected using GLM Lasso and stepwise selection ${ }^{20}$ based on the Akaike Information Criterion. ${ }^{1}$ We further applied other causal inference methods, but because the results were consistent and for the sake of brevity, we did not include them here. To account for baseline blood pressure and heart rate (HR) as potential confounders, we performed a subgroup analysis limited to only those who had these data available (available in $37 \%$ of the subjects) and included blood pressure and HR in the final outcome model. To account for other complications, we also included the same 443 diagnoses present on admission as confounders in the model. Furthermore, to adjust the model by initial GCS score, we used propensity score matching based on a subgroup of patients (40\% of nontraumatic cohort) with at least one record of GCS score prior to the treatment.

\section{Results}

Of 2634 patients with nontraumatic SAH, 559 (21.2\%), $1342(50.9 \%)$, and $733(27.8 \%)$ were initially treated with dopamine, phenylephrine, and norepinephrine, respectively. Baseline demographics, characteristics, mortality, and comorbidities are summarized by the three treatment groups in Table 1.

\section{Baseline Demographics and Vital Signs}

The population included $64 \%$ females and the majority (66\%) were white race. Patients administered dopamine were, on average, slightly older than those in the other two treatment groups $(58.3,56.2$, and 55.7 years old for dopamine, phenylephrine, and norepinephrine groups, respectively; $p=0.003$ ). The phenylephrine group had the largest percentage of whites $(68 \%)$ and the smallest percentage of African Americans (19.7\%) among the three groups ( $p$ $<0.001$ ). The patients in the three treatment groups had significantly different average baseline blood pressures and HRs, where the average HRs were higher in the norepinephrine group, and the average blood pressures were higher in the phenylephrine group, compared to those of other two treatment groups ( $\mathrm{p}<0.001$ and 0.004$)$.

\section{Comorbidities and Outcomes}

Among 2634 nontraumatic SAH patients, 59.8\% were discharged alive, $36.5 \%$ died in the hospital or were discharged to hospice care, and $3.7 \%$ had an unknown mortality status. There was a markedly lower mortality rate in the phenylephrine group compared to the dopamine and norepinephrine groups $(24.5 \%, 50.6 \%$, and $47.6 \%$, respectively; $\mathrm{p}<0.001$; Table 1). The norepinephrine group had a higher burden of comorbidities including CCI, pneumo- 
TABLE 1. Baseline demographics and clinical characteristics/outcomes of nontraumatic aneurysmal SAH patients by vasopressor treatment group

\begin{tabular}{|c|c|c|c|c|c|}
\hline Characteristic & Total & Dopamine & Phenylephrine & Norepinephrine & $p$ Value \\
\hline \multicolumn{6}{|l|}{ Baseline/demographics } \\
\hline No. of patients (\%) & $2634(100)$ & $559(21.2)$ & $1342(50.9)$ & $733(27.8)$ & $<0.001$ \\
\hline Mean age $\pm S D$, yrs & $56.5 \pm 14.7$ & $58.3 \pm 15.4$ & $56.2 \pm 14.3$ & $55.7 \pm 15.0$ & 0.003 \\
\hline Female sex, $\%$ & 63.9 & 65.1 & 63.6 & 63.4 & 0.79 \\
\hline Race, $\%$ & & & & & $<0.001$ \\
\hline White & 66.0 & 66.9 & 68.0 & 61.8 & \\
\hline African American & 22.7 & 22.2 & 19.7 & 28.5 & \\
\hline Other & 11.3 & 10.9 & 12.4 & 9.7 & \\
\hline Mean SBP $\pm \mathrm{SD}^{*}$ & $122.4 \pm 28.9$ & $113.8 \pm 30.7$ & $126.4 \pm 27.2$ & $117.6 \pm 30.1$ & $<0.001$ \\
\hline Mean DBP $\pm S D^{*}$ & $65.4 \pm 16.3$ & $64.3 \pm 17.5$ & $67.0 \pm 15.2$ & $63.2 \pm 17.6$ & 0.004 \\
\hline Mean HR $\pm \mathrm{SD}^{*}$ & $84.3 \pm 21.9$ & $84.4 \pm 24.0$ & $80.4 \pm 18.7$ & $92.0 \pm 24.6$ & $<0.001$ \\
\hline \multicolumn{6}{|l|}{ Outcomes/comorbidities } \\
\hline Mortality, \% & 36.5 & 50.6 & 24.5 & 47.6 & $<0.001$ \\
\hline Mean LOS \pm SD, days $\dagger$ & $17.5 \pm 19.8$ & $13.1 \pm 15$ & $19.2 \pm 15.9$ & $17.9 \pm 27.7$ & $<0.001$ \\
\hline Pneumonia, \% & 22.3 & 16.1 & 22.1 & 29.2 & $<0.001$ \\
\hline Anemia, \% & 20.0 & 17.2 & 18.8 & 24.6 & 0.001 \\
\hline Sepsis, \% & 10.1 & 7.3 & 7.5 & 17.1 & $<0.001$ \\
\hline MI, \% & 6.5 & 7.5 & 4.4 & 9.5 & $<0.001$ \\
\hline Acute renal failure, $\%$ & 9.9 & 8.9 & 6.6 & 17.2 & $<0.001$ \\
\hline Pulmonary edema, $\%$ & 1.3 & 0.4 & 1.2 & 2.0 & 0.03 \\
\hline Heart failure, \% & 9.4 & 9.5 & 7.7 & 12.4 & 0.002 \\
\hline Diabetes, $\%$ & 10.8 & 11.6 & 9.6 & 12.4 & 0.11 \\
\hline Liver disease, $\%$ & 2.1 & 1.4 & 1.5 & 3.5 & 0.003 \\
\hline Mean $\mathrm{CCl} \pm \mathrm{SD}$ & $1.8 \pm 1.0$ & $1.7 \pm 0.9$ & $1.7 \pm 1.0$ & $2 \pm 1.2$ & $<0.001$ \\
\hline \multicolumn{6}{|c|}{$\begin{array}{l}\text { LOS = hospital length of stay. } \\
\text { All } p \text { values are calculated using an ANOVA or Fisher's exact test. } \\
\text { * Baseline blood pressure and heart rate (the last measurement prior to the administration of the first vasopressor) were } \\
\text { available and analyzed for the } 37 \% \text { available subjects. } \\
\text { † For LOS one patient is excluded due to incomplete data; medians are } 15,8,16 \text {, and } 14 \text {, respectively }(p<0.001) \text {. }\end{array}$} \\
\hline
\end{tabular}

TABLE 2. Percentage of patients according to GCS scores in each of the three treatment groups and mortality breakdown by treatment group and GCS categories

\begin{tabular}{lrrrrrr}
\hline & \multicolumn{5}{c}{ GCS Score } & \\
\cline { 2 - 6 } \multicolumn{1}{c}{ Treatment Group } & $3-5$ & $6-8$ & $9-11$ & $12-14$ & 15 & Total \\
\hline Percentage of patients & & & & & & \\
\hline$\quad$ Dopamine & 69.9 & 13.3 & 1.8 & 11.5 & 3.5 & 100 \\
\hline$\quad$ Norepinephrine & 67.6 & 14.3 & 4.1 & 10.2 & 3.8 & 100 \\
\hline$\quad$ Phenylephrine & 48.1 & 19.7 & 10.8 & 17.5 & 4.0 & 100 \\
\hline Mortality rate & & & & & & \\
\hline$\quad$ Dopamine & 73.4 & 40.0 & 50.0 & 15.4 & 25.0 & 60.2 \\
\hline$\quad$ Norepinephrine & 69.5 & 26.7 & 15.4 & 6.3 & 50.0 & 54.0 \\
\hline$\quad$ Phenylephrine & 40.6 & 11.1 & 4.7 & 1.0 & 4.2 & 22.5 \\
\hline Weighted averages & 55.7 & 17.5 & 7.6 & 3.4 & 20.0 & \\
\hline
\end{tabular}

All data given as percentages. nia, anemia, sepsis, myocardial infarction, acute renal failure, and heart failure.

\section{GCS Scores}

Table 2 presents the patient percentages and mortality by treatment group and GCS categories for the $980 \mathrm{pa}-$ tients (40\% of nontraumatic SAH) with at least one GCS record prior to administration of the vasopressors. Patients in the phenylephrine group had more favorable initial GCS scores than those in the dopamine and norepinephrine groups. For example, there was a smaller percentage of patients with GCS scores between 3 and 5 among the phenylephrine group (48.1\%) compared to the dopamine and norepinephrine groups $(69.9 \%$ and $67.6 \%$, respectively). However, when comparing the treatment groups among each subgroup of GCS category, phenylephrine consistently had lower mortality rates. This observation was confirmed in propensity-matched sensitivity analysis below.

\section{Vasopressor Treatment Effects Based on Causal Inference Analysis}

Table 3 summarizes the results from the propen- 
TABLE 3. Association of treatment with mortality using a logistic regression model (unweighted), and propensity adjustment via IPW (weighted) for nontraumatic aneurysmal SAH subjects, adjusted for age, race, marital status, and sex

\begin{tabular}{|c|c|c|c|c|c|}
\hline \multirow[b]{2}{*}{ Comparison } & \multirow[b]{2}{*}{ Deaths/Cohort (\%) } & \multicolumn{2}{|c|}{ Unweighted Model } & \multicolumn{2}{|c|}{ Weighted Model } \\
\hline & & OR $(95 \% \mathrm{Cl})$ & p Value & OR $(95 \% \mathrm{Cl})$ & $\mathrm{p}$ Value \\
\hline \multicolumn{6}{|c|}{ Dopamine vs phenylephrine } \\
\hline Dopamine & $251 / 492(51 \%)$ & $3.02(2.42-3.76)$ & $<0.0001$ & $2.19(1.70-2.81)$ & $<0.001$ \\
\hline Phenylephrine & $318 / 1253(25 \%)$ & Ref & Ref & Ref & Ref \\
\hline \multicolumn{6}{|c|}{ Norepinephrine vs phenylephrine } \\
\hline Norepinephrine & $311 / 672(46 \%)$ & $2.63(2.15-3.22)$ & $<0.0001$ & $2.24(1.80-2.80)$ & $<0.001$ \\
\hline Phenylephrine & $318 / 1253(25 \%)$ & Ref & Ref & Ref & Ref \\
\hline \multicolumn{6}{|c|}{ Dopamine vs norepinephrine } \\
\hline Dopamine & $251 / 492(51 \%)$ & $1.15(0.91-1.45)$ & 0.25 & $0.97(0.75-1.27)$ & 0.85 \\
\hline Norepinephrine & $311 / 672(46 \%)$ & Ref & Ref & Ref & Ref \\
\hline
\end{tabular}

sity score model, and Supplementary Fig. S1 presents a graphic representation of the balance achieved through IPW. The adjusted odds (controlled for age, race, marital status, and sex) for mortality in the dopamine and norepinephrine groups were significantly higher than that for the phenylephrine group, in both unweighted and propensity-weighted models. Specifically, propensity adjustment via IPW modestly attenuated the adjusted odds ratio, but mortality remained increased for both dopamine and norepinephrine compared with phenylephrine (unweighted OR $3.02,95 \%$ CI 2.42-3.76, weighted OR $2.19,95 \%$ CI 1.70-2.81; and unweighted OR 2.63, 95\% CI 2.15-3.22, weighted OR 2.24, 95\% CI 1.80-2.80). Furthermore, there was no significant difference between the odds of mortality for dopamine and norepinephrine (weighted OR 0.97, 95\% CI 0.75-1.27).

\section{Sensitivity Analyses}

The vital sign records are not available in the database prior to 2009, and it is the hospital's decision which tables to report to the Cerner database. Hence, important baseline measurements such as systolic blood pressure (SBP), diastolic blood pressure (DBP), and HR are only available for $37 \%$ of the patients in our cohort. We conducted subgroup analyses using the patients who had available data on baseline DBP, SBP, HR, and potential confounders of the treatment outcome association (Supplementary Tables S2 and S3). Accounting for these measurements did not change the findings. Furthermore, a subgroup analysis based on $40 \%$ of nontraumatic patients with at least one GCS record and matched based on GCS score and demographic variables confirmed the results (OR 4.31, 95\% CI 2.53-7.32 for norepinephrine vs phenylephrine; OR 3.01, 95\% CI 1.07-8.48 for dopamine vs phenylephrine; and OR 0.98, 95\% CI 0.39-2.44 for dopamine vs norepinephrine; Supplementary Table S4). Supplementary Tables S5-S7 show that GCS score and demographic factors were well balanced between each pair of treatments. We confirmed the associations between the initial vasopressor and mortality with several causal inference statistical models. For example, we conducted pairwise treatment analyses by creating separate propensity score models for each group of treatments (i.e., dopamine vs phenylephrine, norepinephrine vs phenylephrine, dopamine vs norepinephrine). We also examined whether the percentage of subjects who died differed by treatment group in the subgroups of subjects who had at least one of three comorbidities: MI, acute renal failure, or sepsis (Supplementary Table S8).

Table 4 summarizes the treatment effect on mortality in a logistic regression with two underlying variable selection approaches: stepwise and LASSO. Although in this study we were primarily interested in the nontraumatic SAH cohort (2634 patients), we repeated the analyses for all SAH patients (4810 patients, including patients with traumatic SAH) and for the subgroup of patients with records for DBP, SBP, and HR, and for those without these vital signs. The results were consistent with our propensity score models. Sensitivity analysis showed the OR of mortality for the dopamine versus phenylephrine group ranged from 1.54 to 2.84 , which contains our reported point estimate from the full sample. No significant difference in the odds of mortality was seen between dopamine and norepinephrine (range 0.86-1.11). The same results were found when we took into account other complications and diagnoses that were present at admission.

\section{Discussion}

Patients who received phenylephrine, dopamine, or norepinephrine as their first or only vasopressor had a mortality rate of $24.5 \%, 50.6 \%$, and $47.6 \%$, respectively (Table 1). The treatment administered to the most patients was phenylephrine (50.9\%). Importantly, the mortality benefit associated with phenylephrine was preserved even when the acute comorbidities of acute MI, renal failure, and sepsis were included.

Our data indicate that phenylephrine administered for nontraumatic SAH is associated with reduced mortality over other vasopressors, which the investigators found surprising due to the sympathomimetic role of phenylephrine in causing vasoconstriction while not increasing cardiac output. ${ }^{22}$ Some research up to this point suggests phenylephrine is not the optimal drug for aneurysmal SAH. In an evaluation by Roy et al., among 63 patients who de- 


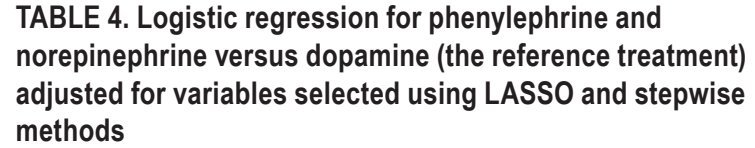

\begin{tabular}{cll}
\hline \multicolumn{1}{c}{ Cohort } & $\begin{array}{c}\text { Dopamine vs } \\
\text { Phenylephrine }\end{array}$ & $\begin{array}{c}\text { Dopamine vs } \\
\text { Norepinephrine }\end{array}$ \\
\hline All SAH patients & & \\
\hline LASSO & $2.00(<0.001)$ & $0.86(0.16)$ \\
\hline All & $2.22(<0.001)$ & $0.88(0.55)$ \\
\hline All w/ BP-HR & $1.78(<0.001)$ & $0.88(0.34)$ \\
\hline All w/o BP-HR & & \\
\hline Stepwise & $1.98(<0.001)$ & $0.86(0.14)$ \\
\hline All & $2.39(<0.001)$ & $0.92(0.7)$ \\
\hline All w/ BP-HR & $1.87(<0.001)$ & $0.89(0.37)$ \\
\hline All w/o BP-HR & & \\
\hline Nontraumatic SAH & & \\
\hline LASSO & $1.79(<0.001)$ & $0.86(0.32)$ \\
\hline Nontraumatic & $2.47(0.004)$ & $1.01(0.98)$ \\
\hline Nontraumatic w/ BP-HR & $1.54(0.007)$ & $0.87(0.46)$ \\
\hline Nontraumatic w/o BP-HR & & \\
\hline Stepwise & $1.85(<0.001)$ & $0.86(0.3)$ \\
\hline Nontraumatic & $2.84(0.001)$ & $1.11(0.74)$ \\
\hline Nontraumatic w/ BP-HR & $1.60(0.003)$ & $0.87(0.44)$ \\
\hline Nontraumatic w/o BP-HR & & \\
\hline BP & & \\
\hline
\end{tabular}

$\mathrm{BP}=$ blood pressure.

For each model the $O R$ ( $p$ value) is given. The analyses are also repeated for all SAH patients, for subgroups of patients with baseline blood pressure and $H R$, and those without baseline blood pressure and $H R$.

veloped DCI associated with SAH, phenylephrine was associated with worse outcomes. ${ }^{32}$ While our population did have a substantial percentage of patients who had a change in vasopressor during the same encounter of care (33\% of nontraumatic SAH patients), for the purposes of our analysis we followed the intention-to-treat approach by categorizing each patient according to their initial vasopressor administered. Interestingly, Joseph et al. analyzed cerebral blood flow using xenon tomography and found that cerebral blood flow increased by $75 \%$ with phenylephrine, although this study did not directly compare phenylephrine to dopamine or norepinephrine. ${ }^{14}$ Similarly, Muizelaar and Becker found increases in cerebral blood flow with phenylephrine administration using xenon tracers. ${ }^{24}$

The study population appears to reflect previous studies assessing SAH management; in fact, the fraction of patients receiving phenylephrine identically matched those reported in 2011 by Meyer et al. when she performed a survey of practicing neurointensivists. ${ }^{21}$ Meyer's survey had an excellent response rate of $45 \%$. Phenylephrine has several attributes that set it apart from norepinephrine and dopamine, including the potential to be given peripherally for many hours safely with appropriate concentration and safety protocols, thereby avoiding potential delays related to hospital policies preventing peripheral administration of dopamine or norepinephrine.? As norepinephrine and dopamine are recognized to be relatively more potent than phenylephrine, their use may potentially lead to blood pressure increases that exceed the endpoint established by the neurocritical care team. Such a phenomenon may lead to complications stemming from excessive hypertension, such as posterior reversible encephalopathy syndrome (PRES). While PRES is noted to be rare, complications from this disease are known to occur and may potentially be unrecognized in routine clinical care. ${ }^{17}$ Phenylephrine has not been associated with changes in intracranial pressure, while dopamine is associated with slightly higher intracranial pressures than norepinephrine in a small clinical study of head trauma patients in which patients were switched between norepinephrine and dopamine. ${ }^{27}$

The trend to refer patients to high-volume centers is well founded and rationalized by the presence of tertiary and experienced multidisciplinary personnel with a full array of interventional capabilities including neuroangiography; the threshold previously described is $>60$ cases per year. ${ }^{35}$ We were not able to precisely validate such findings, although there was a significant trend favoring care in a more experienced center that supports the current health system paradigm (Supplementary Fig. S2).

Limitations to our study include the retrospective nature of the Cerner Health Facts database. While our population is relatively large and representative given the incidence rate of SAH in the US ${ }^{9,34,38}$ and includes granular data of drugs administered, functional status upon discharge (modified Rankin Scale score, etc.) was not available and such outcomes cannot be considered with this data set. Additionally, while we used the new diagnosis of SAH and the concomitant administration of vasopressors to indicate severe disease, we were not able to confirm with imaging whether or not included patients had aneurysmal disease. When adding GCS score to the propensity score model to account for patient severity, IPW did not return a desirable GCS balance among the vasopressor treatment groups; hence, instead of IPW we used propensity scores to match the subjects and obtained satisfactory GCS scores and other covariate balance. Given that this analysis confirmed our primary analyses, we expect that the medication variables and demographics probably captured patient severity and factors associated with mortality. Finally, the important comorbidities that could be confounders of the treatment-mortality relationship have no time of onset information in the database. Therefore, we cannot determine whether comorbidities existed before the administration of vasopressors, or whether they occurred due to the vasopressors or associated complications and could be considered as surrogate outcomes. Hence, we only adjusted for known pretreatment medications (surrogates for comorbidities) and demographics in the propensity model. Due to potential imprecision in available baseline diagnostic variables for use in the propensity models, there is the possibility for unmeasured confounding because the odd ratios become more and more attenuated as the adjustment performed becomes more stringent, but never to null. This could be a sign of unmeasured confounding, violating one of the two fundamental assumptions of propensity scoring. Being able to include more pretreatment clinical covariates in the model would further strengthen the analysis. Future work includes studying the difference between va- 
sopressors compensating for patients who receive multidrug therapy, single drug therapy, and those who do not become ill enough to require vasopressor therapy.

\section{Conclusions}

Dopamine, phenylephrine, and norepinephrine are frequently administered in the setting of high-grade SAH. Phenylephrine administration is associated with a substantial reduction in mortality among these three agents for patients admitted with a new diagnosis of aneurysmal SAH requiring vasopressors. Dopamine usage was associated with the highest mortality. Prospective studies are warranted to further evaluate these findings.

\section{Acknowledgments}

The authors acknowledge the contributions from all members of the EHR Working Group at the Center for Big Data in Health Sciences CBD-HS. This project is supported by the CBD-HS at the School of Public Health, University of Texas Health Science Center at Houston (UTHealth), and partially supported (support on data preparation) by the SBMI Data Service Office and Data Science and Informatics Core for Cancer Research (funded by CPRIT grant no. RP170668) at UTHealth. Some of the trainees are supported by the NIH training grant no. 2T32GM074902. Because of the sensitive nature of the data collected for this study, requests to access the data from qualified researchers trained in human subject confidentiality protocols may be sent to Hulin $\mathrm{Wu}$ at hulin.wu@uth.tmc.edu.

\section{References}

1. Akaike H: A new look at the statistical model identification. IEEE Trans Automat Contr 19:716-723, 1974

2. Austin PC: The relative ability of different propensity score methods to balance measured covariates between treated and untreated subjects in observational studies. Med Decis Making 29:661-677, 2009

3. Austin PC, Stuart EA: Moving towards best practice when using inverse probability of treatment weighting (IPTW) using the propensity score to estimate causal treatment effects in observational studies. Stat Med 34:3661-3679, 2015

4. Budohoski KP, Guilfoyle M, Helmy A, Huuskonen T, Czosnyka M, Kirollos R, et al: The pathophysiology and treatment of delayed cerebral ischaemia following subarachnoid haemorrhage. J Neurol Neurosurg Psychiatry 85:1343-1353, 2014

5. Connolly ES Jr, Rabinstein AA, Carhuapoma JR, Derdeyn CP, Dion J, Higashida RT, et al: Guidelines for the management of aneurysmal subarachnoid hemorrhage: a guideline for healthcare professionals from the American Heart Association/American Stroke Association. Stroke 43:1711-1737, 2012

6. Crispo JAG, Willis AW, Thibault DP, Fortin Y, Hays HD, McNair DS, et al: Associations between anticholinergic burden and adverse health outcomes in Parkinson disease. PLoS One 11:e0150621, 2016

7. Delgado T, Wolfe B, Davis G, Ansari S: Safety of peripheral administration of phenylephrine in a neurologic intensive care unit: a pilot study. J Crit Care 34:107-110, 2016

8. Diringer MN, Bleck TP, Claude Hemphill J III, Menon D, Shutter L, Vespa P, et al: Critical care management of patients following aneurysmal subarachnoid hemorrhage: recommendations from the Neurocritical Care Society's Multidisciplinary Consensus Conference. Neurocrit Care 15:211-240, 2011

9. Etminan N, Chang HS, Hackenberg K, de Rooij NK, Ver- gouwen MDI, Rinkel GJE, et al: Worldwide incidence of aneurysmal subarachnoid hemorrhage according to region, time period, blood pressure, and smoking prevalence in the population: a systematic review and meta-analysis. JAMA Neurol 76:588-597, 2019

10. Feng P, Zhou XH, Zou QM, Fan MY, Li XS: Generalized propensity score for estimating the average treatment effect of multiple treatments. Stat Med 31:681-697, 2012

11. Fernando SM, Perry JJ: Subarachnoid hemorrhage. CMAJ 189:E1421, 2017

12. Francoeur CL, Mayer SA: Management of delayed cerebral ischemia after subarachnoid hemorrhage. Crit Care 20:277, 2016

13. Imbens GW: The role of the propensity score in estimating dose-response functions. Biometrika 87:706-710, 2000

14. Joseph M, Ziadi S, Nates J, Dannenbaum M, Malkoff M: Increases in cardiac output can reverse flow deficits from vasospasm independent of blood pressure: a study using xenon computed tomographic measurement of cerebral blood flow. Neurosurgery 53:1044-1052, 2003

15. Kosnik EJ, Hunt WE: Postoperative hypertension in the management of patients with intracranial arterial aneurysms. J Neurosurg 45:148-154, 1976

16. Lin CL, Dumont AS, Zhang JH, Zuccarello M, Muroi C: Cerebral vasospasm after aneurysmal subarachnoid hemorrhage: mechanism and therapies. Biomed Res Int 2014:2014, 679014

17. Madaelil TP, Dhar R: Posterior reversible encephalopathy syndrome with thalamic involvement during vasopressor treatment of vertebrobasilar vasospasm after subarachnoid hemorrhage. J Neurointerv Surg 8:e45, 2016

18. Mayberg MR, Batjer HH, Dacey R, Diringer M, Haley EC, Heros RC, et al: Guidelines for the management of aneurysmal subarachnoid hemorrhage. A statement for healthcare professionals from a special writing group of the Stroke Council, American Heart Association. Stroke 25:2315-2328, 1994

19. McCaffrey DF, Griffin BA, Almirall D, Slaughter ME, Ramchand R, Burgette LF: A tutorial on propensity score estimation for multiple treatments using generalized boosted models. Stat Med 32:3388-3414, 2013

20. Menard S: Logistic Regression: From Introductory to Advanced Concepts and Applications. Thousand Oaks, CA: SAGE Publications, 2014

21. Meyer R, Deem S, Yanez ND, Souter M, Lam A, Treggiari MM: Current practices of triple-H prophylaxis and therapy in patients with subarachnoid hemorrhage. Neurocrit Care 14:24-36, 2011

22. Miller JA, Dacey RG Jr, Diringer MN: Safety of hypertensive hypervolemic therapy with phenylephrine in the treatment of delayed ischemic deficits after subarachnoid hemorrhage. Stroke 26:2260-2266, 1995

23. Muehlschlegel S, Dunser MW, Gabrielli A, Wenzel V, Layon AJ: Arginine vasopressin as a supplementary vasopressor in refractory hypertensive, hypervolemic, hemodilutional therapy in subarachnoid hemorrhage. Neurocrit Care 6:3-10, 2007

24. Muizelaar JP, Becker DP: Induced hypertension for the treatment of cerebral ischemia after subarachnoid hemorrhage. Direct effect on cerebral blood flow. Surg Neurol 25:317325, 1986

25. Oshiro EM, Walter KA, Piantadosi S, Witham TF, Tamargo RJ: A new subarachnoid hemorrhage grading system based on the Glasgow Coma Scale: a comparison with the Hunt and Hess and World Federation of Neurological Surgeons Scales in a clinical series. Neurosurgery 41:140-148, 1997

26. Park MY, Hastie T: L1-regularization path algorithm for generalized linear models. J R Stat Soc Series B Stat Methodol 69:659-677, 2007 
27. Ract C, Vigué B: Comparison of the cerebral effects of dopamine and norepinephrine in severely head-injured patients. Intensive Care Med 27:101-106, 2001

28. Rasmy L, Wu Y, Wang N, Geng X, Zheng WJ, Wang F, et al: A study of generalizability of recurrent neural networkbased predictive models for heart failure onset risk using a large and heterogeneous EHR data set. J Biomed Inform 84:11-16, 2018

29. Reynolds MR, Buckley RT, Indrakanti SS, Turkmani AH, Oh G, Crobeddu E, et al: The safety of vasopressor-induced hypertension in subarachnoid hemorrhage patients with coexisting unruptured, unprotected intracranial aneurysms. J Neurosurg 123:862-871, 2015

30. Rose JC, Mayer SA: Optimizing blood pressure in neurological emergencies. Neurocrit Care 1:287-299, 2004 (Erratum in Neurocrit Care 4:98, 2006)

31. Rosenbaum PR: Rubin DB: The central role of the propensity score in observational studies for causal effects. Biometrika 70:41-55, 1983

32. Roy B, McCullough LD, Dhar R, Grady J, Wang YB, Brown RJ: Comparison of initial vasopressors used for delayed cerebral ischemia after aneurysmal subarachnoid hemorrhage. Cerebrovasc Dis 43:266-271, 2017

33. Sakr Y, Dünisch P, Santos C, Matthes L, Zeidan M, Reinhart $\mathrm{K}$, et al: Poor outcome is associated with less negative fluid balance in patients with aneurysmal subarachnoid hemorrhage treated with prophylactic vasopressor-induced hypertension. Ann Intensive Care 6:25, 2016

34. Shea AM, Reed SD, Curtis LH, Alexander MJ, Villani JJ, Schulman KA: Characteristics of nontraumatic subarachnoid hemorrhage in the United States in 2003. Neurosurgery 61:1131-1138, 2007

35. Vespa P, Diringer MN: High-volume centers. Neurocrit Care 15:369-372, 2011

36. Worthington JM, Goumas C, Jalaludin B, Gattellari M: Decreasing risk of fatal subarachnoid hemorrhage and other epidemiological trends in the era of coiling implementation in Australia. Front Neurol 8:424, 2017

37. Yao Z, Hu X, Ma L, You C, He M: Timing of surgery for aneurysmal subarachnoid hemorrhage: a systematic review and meta-analysis. Int J Surg 48:266-274, 2017

38. Zacharia BE, Hickman ZL, Grobelny BT, DeRosa P, Kotchetkov I, Ducruet AF, et al: Epidemiology of aneurysmal subarachnoid hemorrhage. Neurosurg Clin N Am 21:221-233, 2010

\section{Disclosures}

The authors report no conflict of interest concerning the materials or methods used in this study or the findings specified in this paper.

\section{Author Contributions}

Conception and design: Wu, Williams, Maroufy, Rasmy, Yamal, Aguilar. Acquisition of data: Rasmy. Analysis and interpretation of data: Wu, Williams, Maroufy, Rasmy, Brown, Yu, Talebi, Yamal, Aguilar. Drafting the article: Wu, Williams, Maroufy, Rasmy, Yamal, Aguilar. Critically revising the article: Wu, Williams, Maroufy, Rasmy, Yaseen, Miao, Leon Novelo, Zhi, DeSantis, Hongjian Zhu, Yamal, Aguilar. Reviewed submitted version of manuscript: Wu, Williams, Maroufy, Rasmy, Brown, Yamal, Aguilar. Approved the final version of the manuscript on behalf of all authors: Wu. Statistical analysis: $\mathrm{Wu}$, Maroufy, Brown, Yu, Hai Zhu, Wang, Thomas, G Zhu, Yamal. Administrative/technical/material support: Wu, Williams. Study supervision: Wu, Williams, Maroufy, Rasmy, Yamal, Aguilar.

\section{Supplemental Information} Online-Only Content

Supplemental material is available online.

Supplementary Tables and Figures. https://thejns.org/doi/ suppl/10.3171/2020.2.FOCUS191002.

\section{Previous Presentations}

A poster concerning our preliminary findings was presented at the American Society of Anesthesiologists Annual Meeting in San Francisco, California, October 16, 2018.

\section{Correspondence}

Hulin Wu: The University of Texas School of Public Health, Houston, TX.hulin.wu@uth.tmc.edu. 